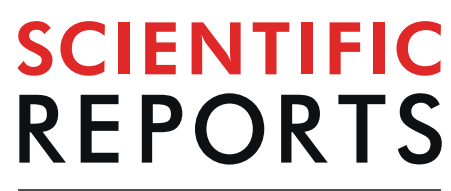

natureresearch

Check for updates

\title{
Giant magnetoresistive biosensors for real-time quantitative detection of protease activity
}

\author{
Sandeep Adem ${ }^{1}$, Sonal Jain ${ }^{1}$, Michael Sveiven ${ }^{1}$, Xiahan Zhou ${ }^{3}$, Anthony J. O’Donoghue ${ }^{2 \bowtie} \&$ \\ Drew A. Hall ${ }^{1,3 凶}$
}

Proteases are enzymes that cleave proteins and are crucial to physiological processes such as digestion, blood clotting, and wound healing. Unregulated protease activity is a biomarker of several human diseases. Synthetic peptides that are selectively hydrolyzed by a protease of interest can be used as reporter substrates of unregulated protease activity. We developed an activity-based protease sensor by immobilizing magnetic nanoparticles (MNPs) to the surface of a giant magnetoresistive spin-valve (GMR SV) sensor using peptides. Cleavage of these peptides by a protease releases the magnetic nanoparticles resulting in a time-dependent change in the local magnetic field. Using this approach, we detected a significant release of MNPs after 3.5 minutes incubation using just $4 \mathrm{nM}$ of the cysteine protease, papain. In addition, we show that proteases in healthy human urine do not release the MNPs, however addition of $20 \mathrm{nM}$ of papain to the urine samples resulted in a time-dependent change in magnetoresistance. This study lays the foundation for using GMR SV sensors as a platform for real-time, quantitative detection of protease activity in biological fluids.

Proteases play an important role in various physiological activities such as food digestion ${ }^{1}$, wound healing ${ }^{2}$, immune function ${ }^{3}$, and intracellular protein turnover ${ }^{4}$. These enzymes cleave between amino acids in proteins and peptides and are the largest class of post-translational modifying enzymes in the human proteome ${ }^{5}$. In cancer and neurodegeneration, unregulated proteolysis can occur when excess proteases are present at the site of disease or alternatively when the endogenous inhibitors are lacking ${ }^{6,7}$. Detection and quantitation of proteases in biofluids can provide a greater understanding of diagnosis and staging of these diseases ${ }^{8,9}$. For example, increased levels of the prostate-specific antigen (PSA) protease in blood is correlated with prostate cancer. This protease is quantified by an enzyme-linked-immunosorbent assay (ELISA) ${ }^{10}$. However, since proteases are catalytically active, there is considerable interest in quantifying enzyme activity rather than protein levels ${ }^{11,12}$. Recently, Ivry and colleagues showed that activity from two aspartic acid proteases, gastricin and cathepsin E, is significantly increased in pre-malignant pancreatic cyst fluid when compared to benign cyst fluid ${ }^{13}$. Development of an assay to screen patient-derived cyst fluid for these protease activities has the potential to stratify patients for surgical intervention or surveillance. In addition, high levels of protease activity occurs in sputum of patients with chronic obstructive pulmonary disease ${ }^{14,15}$, cystic fibrosis ${ }^{16-18}$, and in non-healing wounds ${ }^{19,20}$. Many notable studies have been carried out to detect cathepsin-B and matrix metalloproteinases (MMPs), both involved in cancer and tumor metastasis, using nanoparticles ${ }^{21,22}$, fluorescent tomographic imaging ${ }^{23}$, and droplet microfluidics $^{24}$. A nanoparticle-based approach ${ }^{25}$ was also used to detect the serine protease granzyme $\mathrm{B}$, as a marker of early allograft rejection. Rapid and accurate quantification of proteases in biofluids such as plasma, urine, sputum, saliva, and wound fluid will allow clinicians to make important decisions about the treatment regimen.

To develop an activity-based protease biomarker of disease, it is essential to utilize a substrate that is efficiently cleaved by the protease of interest. Protease assays can be categorized as either homogenous or heterogeneous assays $^{26}$. In a homogenous assay, the substrate and enzyme are both present in solution and generally utilize fluorescent or colorimetric peptide substrates that are illuminated upon protease cleavage ${ }^{27,28}$. In a heterogeneous assay, the enzyme is in solution while the substrate is immobilized. Heterogeneous protease assays have utilized electrochemical detection ${ }^{29,30}$, surface plasmon resonance (SPR) ${ }^{31}$, surface-enhanced Raman spectroscopy

\footnotetext{
${ }^{1}$ University of California - San Diego, Department of Bioengineering, La Jolla, CA, 92093, USA. ${ }^{2}$ University of California - San Diego, Skaggs School of Pharmacy and Pharmaceutical Sciences, La Jolla, CA, 92093, USA. ${ }^{3}$ University of California - San Diego, Department of Electrical and Computer Engineering, La Jolla, CA, 92093, USA.

凶e-mail: ajodonoghue@ucsd.edu; drewhall@ucsd.edu
} 


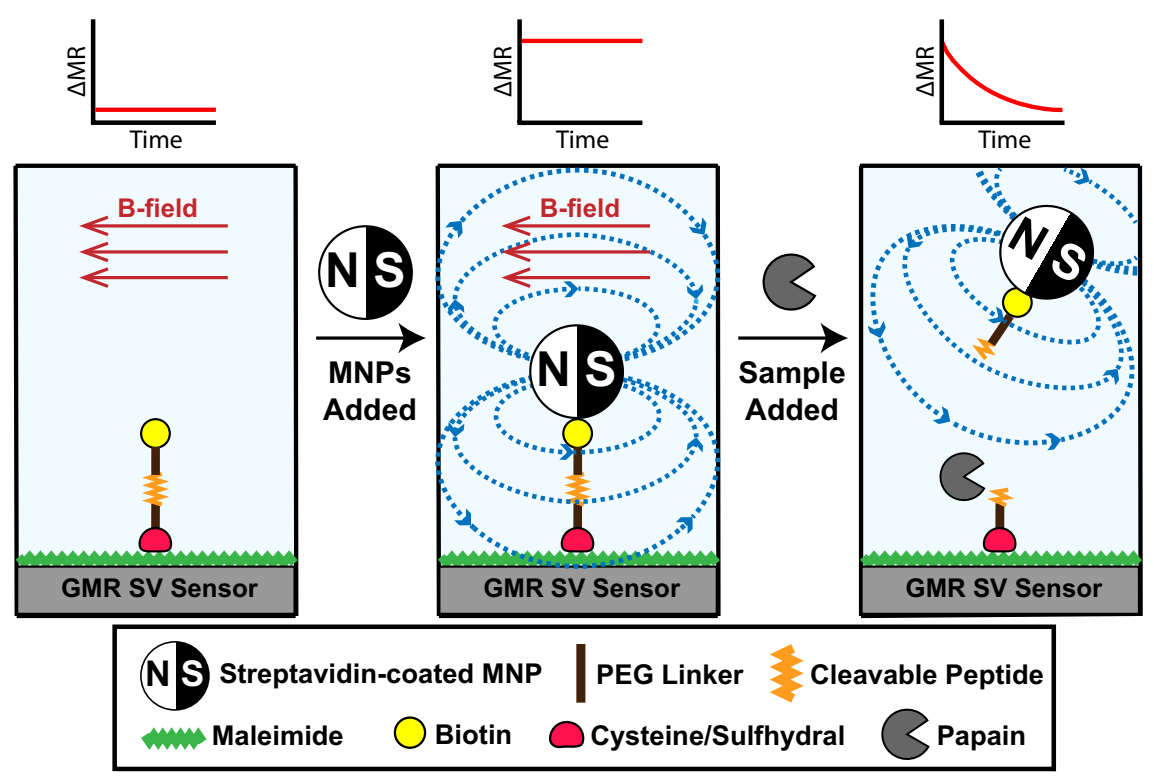

Figure 1. Illustration of the magnetic detection scheme for protease activity. A biotinylated peptide is immobilized on the GMR SV sensors and placed in a magnetic field. Addition of streptavidin-coated MNPs causes an increase in magnetoresistance (MR) as they are orientated close to the sensor surface via the streptavidin-biotin interaction. When a biofluid sample containing a protease is added, cleavage of the peptide causes a time-dependent change in the MR as the MNPs are enzymatically released from the sensor surface.

$(\mathrm{SERS})^{32}$, ELISA, and liquid crystal technology $\mathrm{y}^{33}$ and these assays have been developed for detection of enzyme activity for trypsin, caspase-3, and $\mathrm{MMPs}^{26}$.

In this study, we developed a heterogeneous protease assay that uses giant magnetoresistive spin-valve (GMR SV) sensors. These sensors transduce changes in the local magnetic field into electrical signals and have been used as the read head in hard disk drives ${ }^{34,35}$, current sensors ${ }^{36,37}$, magnetic memory ${ }^{38}$ and biosensors ${ }^{39,40}$. Their operation is rooted in quantum mechanics, exhibiting a phenomenon known as spin-dependent scattering ${ }^{41}$, wherein the device's resistance is proportional to the magnetic field. These sensors are highly scalable, can be produced at low $\operatorname{cost}^{42}$, and manufactured in high volume. There are several advantages to using magnetic-based sensors for a protease assay over homogenous and other heterogeneous assay formats. First, clinical samples do not contain any magnetic content. Therefore, these samples have intrinsically low background signal, enabling high sensitivity. In comparison, optical assays face problems such as autofluorescence ${ }^{43}$ and label-bleaching ${ }^{44}$ that produce an undesired background signal. Second, the GMR SV sensors can be arrayed for multiplexed detection in a single assay without the need for optical scanning. Third, magnetic sensors are insensitive to the sample matrix, allowing them to be used with a wide variety of samples with minimal sample preparation ${ }^{45}$, making them convenient for use in point-of-care (POC) or point-of-use (POU) settings ${ }^{46}$. Lastly, the sensors continuously quantify the local magnetic field changes enabling real-time monitoring of the assay.

In previous applications of GMR SV biosensor technology, recruitment of magnetic nanoparticles (MNPs) to the surface via an antibody-antigen interaction was quantified in real-time ${ }^{47}$. However, in this study, we use this technology in reverse and without the use of antigens or antibodies. MNPs are first tethered to the magnetic sensor surface using a peptide, therefore creating an environment with high magnetoresistance (MR) signal. The peptide sequence is designed to be a substrate for the cysteine protease, papain. Upon addition of this enzyme, the peptide sequence is hydrolyzed, causing the MNPs to be released from the proximity of the sensor (Fig. 1). This results in a decrease in MR that can be monitored in real-time. When a fixed amount of peptide-MNP substrate is present, the release rate correlates with the protease concentration.

\section{Results}

Design of papain peptide substrate for heterogeneous assay. Papain is a well characterized cysteine protease related to several disease-associated proteases such as cathepsin $\mathrm{K}^{48}$ and cruzain ${ }^{49}$. This enzyme is optimally active between pH 5.5 and 7.5 and irreversibly inactivated by the epoxide inhibitor, E- $64^{50}$. We used this enzyme as a model system to test and validate our proof-of-concept heterogeneous GMR SV based protease assay. To identify a peptide sequence that is efficiently cleaved by papain, the enzyme was previously combined with an equimolar mixture of synthetic tetradecapeptides and cleavage products were quantified by mass spectrome$\operatorname{try}^{51}$. Using these data, we identified a tetradecapeptide substrate, KWLIHPTFSYnRWP, that was rapidly cleaved between S-Y and Y-n, where lowercase'n' corresponds to the non-natural amino acid, norleucine. We synthesized the $C$-terminal region of this peptide that contained the sequence TFSYnRWP, corresponding to the amino acids that surround the cleavage sites. However, to make this sequence compatible with the heterogeneous assay format, Biotin- $\mathrm{PEG}_{36}$ and $\mathrm{PEG}_{12}$-Cys were included on the $\mathrm{N}$-terminus and $\mathrm{C}$-terminus, respectively (Fig. 2a). The function of the $C$-terminal cysteine residue is to facilitate immobilization of the Biotin-PEG ${ }_{36}-$ TFSYnRWP-PEG $12-C y s$ 
a)

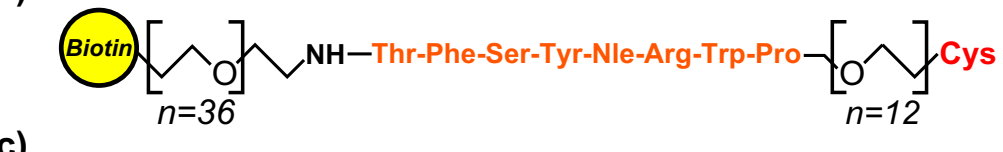

c)

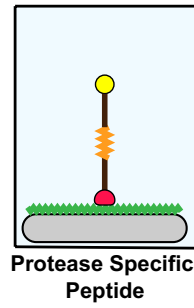

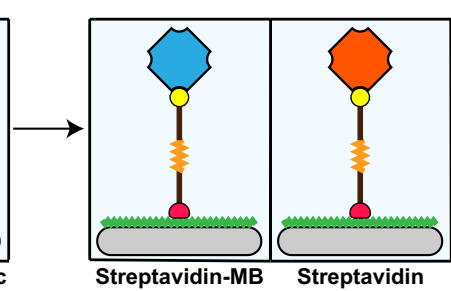

Streptavidin-MB

Streptavidin

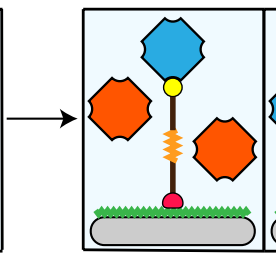

MB => Strep

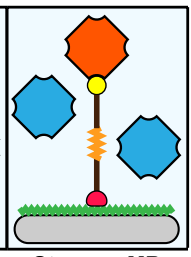

Strep $=>$ MB b)

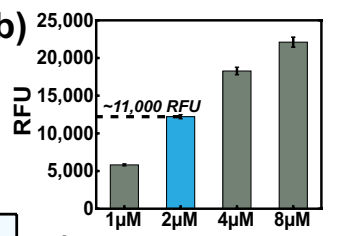

d) \begin{tabular}{rl|l|}
100 \\
90 \\
80
\end{tabular}

Figure 2. (a) Design of a peptide substrate for a papain heterogeneous assay. Amino acids are listed using the standard three-letter code. (b) Standard curves for peptide loading capacity. A concentration of $2 \mu \mathrm{M}$ was used for subsequent optical assays yielding an RFU reading of $\sim 11,000$. (c) Illustration of peptide immobilization scheme and streptavidin loading/blocking on polystyrene plates. (d) Bar graphs comparing the loading of fluorescent streptavidin Marina Blue (blue) and blocking with non-fluorescent streptavidin (orange) to immobilized peptide substrate. Error bars are $\pm 1 \sigma$.

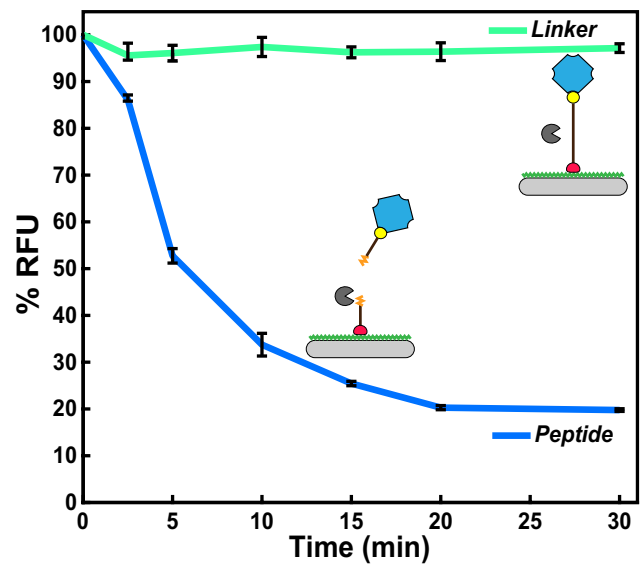

Figure 3. Time-dependent hydrolysis of immobilized peptide substrate by papain. No cleavage of the PEG linker was detected under the same conditions. Error bars are $\pm 1 \sigma$.

molecule (hereafter referred to as "peptide") to a maleimide coated surface through a covalent thioether linkage. The function of the $\mathrm{N}$-terminal biotin group is to bind a streptavidin coated reporter element such as streptavidin-marina blue (SA-MB) or streptavidin-coated MNP (SA-MNP).

Immobilization of papain peptide substrate. The papain substrate (1-8 $\mu \mathrm{M})$ was incubated for 2 hours in a microplate that was functionalized with BSA-maleimide. Unbound peptide was removed by washing and unreacted maleimide was blocked by the addition of free cysteine. The immobilized peptide containing an $\mathrm{N}$-terminal biotin group was then quantified by labeling with SA-MB. We showed that the concentration of peptide directly correlated with the fluorescent signal (Fig. 2b) and we chose a peptide concentration of $2 \mu \mathrm{M}$ for the remainder of our studies as the signal-to-noise ratio (SNR) was greater than 10 .

To ensure that labelling of the immobilized peptide occurs through direct interaction between biotin and streptavidin, a competition assay was performed using SA-MB and non-fluorescent streptavidin (Fig. 2c). When the immobilized papain substrate was first incubated with SA-MB and then with streptavidin, a strong fluorescent signal was detected (Fig. 2d). However, when the peptide substrate was first incubated with non-fluorescent streptavidin followed by SA-MB, no fluorescent signal was detected. These data show that the fluorescent signal generated using this heterogeneous assay is due to direct interaction between SA-MB and the immobilized biotin. In addition, these studies show that binding of the SA-MB complex is stable, as the fluorescent signal cannot be reduced even after 1-hour incubation with non-fluorescent streptavidin.

Papain cleavage of immobilized peptide. After successful peptide loading, cleavage of this sequence by papain and release of SA-MB were evaluated. We incubated the immobilized peptide-SA-MB complex with $20 \mathrm{nM}$ of papain. At defined time intervals between 2.5 and 30 minutes, the enzyme activity was terminated by 
a)

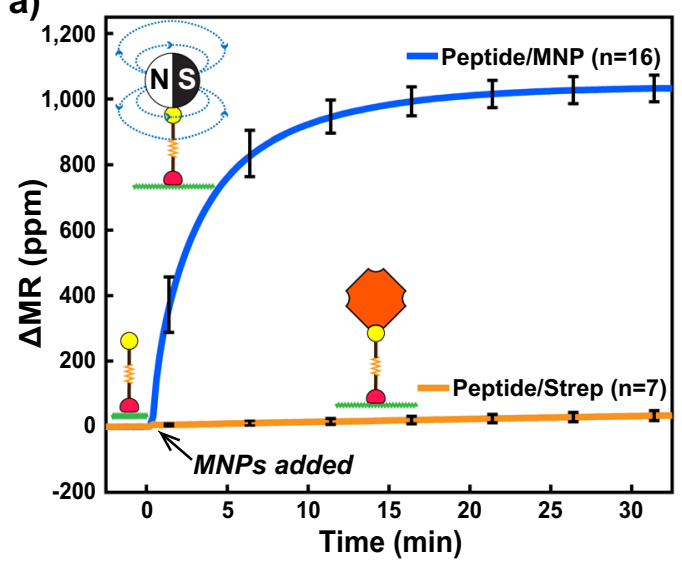

b)

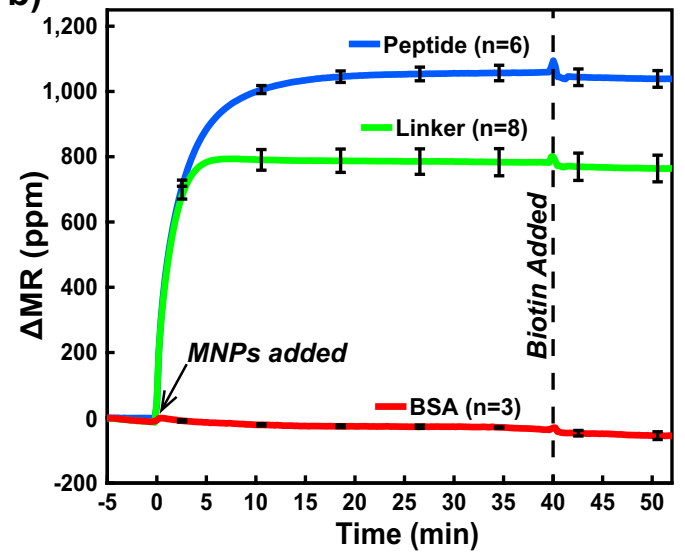

Figure 4. Real-time magnetometry loading data. (a) Loading of MNPs to immobilized peptides that were blocked with streptavidin (orange) or had no blocking (blue). (b) MNP loading to sensors via immobilized peptide (blue) and linker (green). Sensors lacking peptide and linker contained only BSA (red). Curves depicted are the mean signal of sensors and $\mathrm{n}$ corresponds to number of sensors that were functionalized. After 40 minutes, all sensors were incubated with biotin to block all available streptavidin sites on MNPs. Error bars are $\pm 1 \sigma$ and $\Delta \mathrm{MR}$ is the change in magnetoresistance from the initial value $\left(\mathrm{MR}_{0}\right)$ in parts-per-million (ppm).

adding E-64 (Fig. 3). When all time points were acquired, the wells on the microplate were washed to remove peptide-SA-MB that had been enzymatically released from the surface. Any peptide-SA-MB that was still tethered to the surface was then quantified on the fluorescent microplate reader. Enzymatic activity by papain resulted a time-dependent decrease in fluorescence. After only 5 minutes, the fluorescent signal was reduced by $\sim 50 \%$ and an additional $30 \%$ of peptide-SA-MB was removed over the next 15 minutes. The reaction was monitored for an additional 10 minutes ( 30 minutes total), however there was no further change in fluorescence during this time.

To ensure that the time-dependent reduction in fluorescence was due to the enzymatic activity of papain, a biotin- $\mathrm{PEG}_{75}$-thiol (hereafter known as "linker") was included as a control. This linker lacks peptide bonds and therefore cannot be cleaved by papain; however, it can be immobilized to the plate surface via a covalent thioether linkage and bind to SA-MB. We show that papain was unable to reduce the fluorescence signal in wells that contained this linker, therefore confirming that papain cleavage of the peptide causes the release of SA-MB in this heterogeneous assay.

Immobilization of peptide substrate to GMR SV sensor. After verifying cleavage of the immobilized peptide by papain, the assay was translated to the GMR SV sensor array to demonstrate its potential for use in a wash-free, real-time, heterogeneous assay. The biochips consist of an $8 \times 10$ array of GMR SV sensors each measuring $90 \mu \mathrm{m}^{2}$ and are coated with a $50 \mathrm{~nm} \mathrm{SiO}$ 2 passivation layer to protect them from corrosion ${ }^{47}$. The peptide was first conjugated to BSA-maleimide and this complex was nanospotted onto 25 sensors on the biochip. The biochip was placed into a custom designed measurement station that can detect changes in MR (Fig. $\mathrm{S} 1$ ). SA-MNPs containing multiple $\mathrm{Fe}_{2} \mathrm{O}_{3}$ cores embedded in a dextran matrix were added. At this size scale, the MNPs are superparamagnetic, and we predicted that when orientated in close proximity to the sensor surface $(<200 \mathrm{~nm})$ an increase in MR will occur from the stray field generated by the MNPs.

SA-MNPs were added to the biochip and binding to the biotinylated peptide was monitored in real-time for 32.5 minutes. The change in MR on the peptide spotted sensors $(n=16)$ was rapid in the initial 5-min interval $(153.6 \mathrm{ppm} / \mathrm{min}$ ) and then slowed over the remaining $25 \mathrm{~min}$, from $31.0 \mathrm{ppm} / \mathrm{min}$ (between $5-10 \mathrm{~min}$ ) to 1.34 $\mathrm{ppm} / \mathrm{min}$ (from 25-30 min) (Fig. 4a). Streptavidin was pre-incubated with 7 sensors on the biochip, prior to addition of SA-MNPs. Under these conditions, no change in MR occurred. These data show that the change in MR is due to direct binding of SA-MNPs to the immobilized peptide molecule. This assay format can clearly distinguish between bound and unbound SA-MNPs and therefore will enable us to perform protease-mediated MNP release assays.

Additional control conditions were also evaluated on the GMR SV sensors. We showed that the thiol-PEG ${ }_{75}$-biotin linker can be used to immobilize SA-MNPs to the biochip thereby providing us with a non-cleavable control for papain activity assays (Fig. 4b). After 40 minutes of SA-MNPs loading, the GMR SV sensors were incubated with excess biotin to block all available streptavidin molecules on the MNP surface. No MNPs were released from the surface indicating that there was strong affinity between the immobilized biotin and streptavidin on the MNPs (Fig. 4b). This blocking step was predicted to be important for downstream papain digestion assays, as SA-MNPs released by the action of the protease would be unable to re-attach to the surface.

GMR SV-based protease assays. A sensor array containing immobilized SA-MNPs was incubated with $20 \mathrm{nM}$ of papain for 160 minutes and the MNPs were released from the surface in a time-dependent manner. After $160 \mathrm{~min}$ utes, $45 \%$ of the signal was reduced without any washing-steps being performed at pH 7.4 (Fig. $5 \mathrm{a}$ ). Under the same 

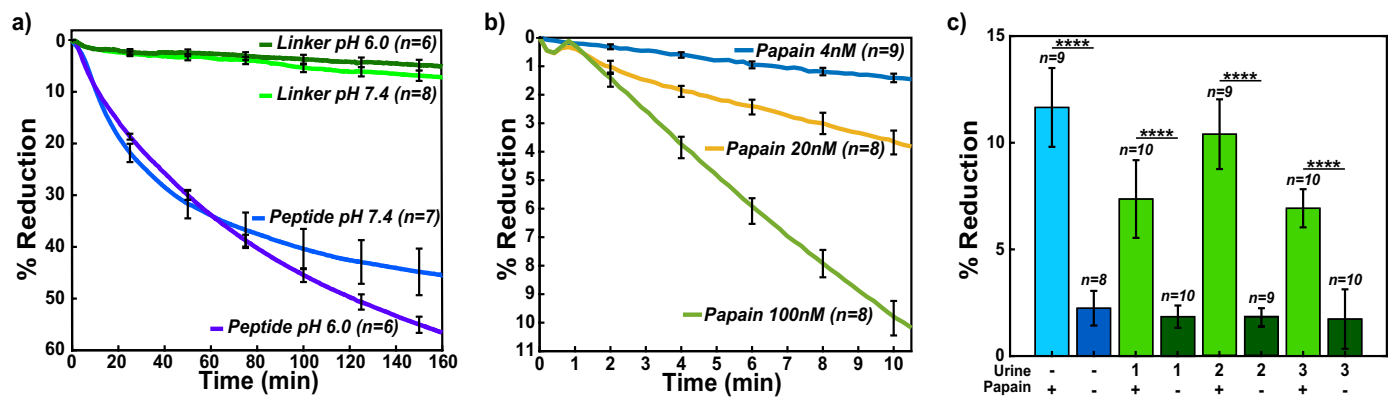

Figure 5. Measured real-time magnetometry papain digestion. (a) Normalized \% reduction of peptide and linker sensors treated with $20 \mathrm{nM}$ papain in $\mathrm{pH} 6.0$ and $\mathrm{pH} 7.4$ conditions. (b) Normalized \% reduction data comparing the $4 \mathrm{nM}$ (blue), $20 \mathrm{nM}$ (yellow), and $100 \mathrm{nM}$ (green) at pH 7.4 peptide treated sensors. Curves depicted are the mean signal of sensors that were functionalized. (c) Normalized \% reduction of papain spiked urine samples and PBS. Error bars are $\pm 1 \sigma$.

conditions, sensors containing the non-cleavable linker sequence, showed only 6\% reduction in MNP signal. These studies confirmed that protease activity can be measured in real-time using a wash-free GMR SV sensor assay.

For a magnetic nanosensor protease assay to have utility in a POC or POU setting, it should be able to rapidly quantify the protease concentration in a biofluid sample under a variety of assay conditions. Biofluids such as plasma, sputum, and wound fluid have a $\mathrm{pH}$ value close to neutral, and therefore the prototype assay described above is suitable for detecting protease activity under these conditions. However, other biofluids such as urine have a $\mathrm{pH}$ of $6.0^{52}$, and it was unclear if the assay was compatible with these mildly acidic conditions. Papain is enzymatically active at pH 6.0 and therefore we evaluated the magnetic nanosensor assay in these conditions. We show that $57 \%$ of the MNPs are released from the sensor surface after 160 minutes incubation with $20 \mathrm{nM}$ of papain at pH 6.0 while only $4 \%$ of the linker is released (Fig. 5a). Papain is more stable at acidic $\mathrm{pH}$ than at neutral $\mathrm{pH}$ and therefore the greater release of MNPs at $\mathrm{pH} 6.0$ relative to $\mathrm{pH} 7.4$ is likely due to increased stability of the enzyme in the acidic environment.

Next, we determined if the rate of MNP release correlates with enzyme concentration and if sufficient enzyme activity data can be generated within a shorter assay time. To do this, we assayed peptide and MNP coated biochips with 4,20 , and $100 \mathrm{nM}$ of papain for 10 minutes. The MNP release from the surface strongly correlates with the concentration of enzyme in the assay (Fig. 5b). Therefore, the rate of change in MR can be used to quantify the amount of papain in the assay. The minimum assay time where protease cleavage can be quantified corresponds to the time when the SNR is greater than 2. Under these conditions, $4 \mathrm{nM}$ of papain was detected after 3.49 minutes incubation while $100 \mathrm{nM}$ of papain can be quantified after 2.87 minutes incubation. These data validate the use of GMR SV sensors to rapidly quantify protease activity using a peptide release assay.

Papain activity in urine. Urine is a commonly used biofluid for diagnosis of urinary tract infections caused by either bacteria or yeast ${ }^{53}$. Proteomic studies have shown that at least 41 proteases are present in urine from healthy individuals and fluorescent reporter peptides have been used to detect activity from these enzymes ${ }^{54}$. The most abundant urine proteases prefer cleaving one or two amino acids from the free $N$-terminus of proteins and peptides and therefore we predicted that these enzymes would not cleave the Biotin-PEG ${ }_{36}$-TFSYnRWP-PEG 12 -Cys peptide because the $N$-terminal threonine (T) residue is coupled to PEG $_{36}$ and therefore blocked. Urine also contains a broad acting cysteine protease inhibitor, cystatin $\mathrm{C}$ that potently inhibits papain. Using urine from three healthy individuals, cystatin $\mathrm{C}$ was inactivated with human cathepsin B. When these urine samples were then added to the GMR SV sensor containing the peptide and incubated for 10 minutes, no significant reduction of MNPs was detected. This study shows that cathepsin B and the endogenous urine proteases are unable to cleave the papain peptide substrate. Addition of $20 \mathrm{nM}$ papain to these urine samples for 10 minutes resulted in a reduction in MR that was comparable to papain assayed in PBS (Fig. 5c). The average time for the SNR to be greater than 2 in these samples was $3.76 \pm 0.25$ minutes.

\section{Discussion}

The goal of this research is to demonstrate that GMR SV sensor-based detection can provide a POC or POU platform to quantify proteases in patient samples. We show that the rate of MNP release by papain directly correlates with the concentration of papain in the assay. Therefore, this platform technology can utilize protease activity in a biological sample as a proxy for quantifying the concentration of the enzyme. Importantly, papain does not release MNPs when these particles are tethered to the sensor surface by a non-peptide linker. In addition, we show that a protease-containing sample such as urine, does not release MNPs when the peptide substrate was designed for cleavage by papain. However, upon addition of papain to the urine, the MNPs are released at a similar rate to reactions that contain just papain in assay buffer.

In the described assay set-up, any peptide substrate that is cleaved by a protease-of-interest can be used in this magnetic sensor assay. The current GMR SV chips contain 80 sensors allowing for quantitation of multiple proteases in the same biofluid sample if they cleave different peptide substrates. Previous studies have shown that there is prevalence of proteinases secreted by pathogenic strains of Proteus bacteria and a significant increase in protease activity in urine of patients with urinary tract infections ${ }^{55,56}$. Our studies demonstrate the ability to 


\begin{tabular}{|c|c|c|c|c|c|c|}
\hline Detection principle & Protease & Buffer/Biofluid & Sensitivity & Assay time & Protease Enrichment & \begin{tabular}{|l} 
Sample \\
Prep
\end{tabular} \\
\hline Colorimetric $^{71}$ & Neutrophil elastase, MMPs & wound fluid & N/A & $15 \mathrm{~min}$ & No & No \\
\hline Colorimetric $^{72}$ & Neutrophil elastase & sputum & $338 \mathrm{nM}$ & $10 \mathrm{~min}$ & Inhibitor capture & Yes \\
\hline Colorometric $^{78}$ & Trypsin & carbonate buffer & $400 \mathrm{pM}$ & $3.5 \mathrm{hr}$ & No & No \\
\hline Electrochemical $^{29}$ & Trypsin & serum & $600 \mathrm{pM}$ & $30 \mathrm{~min}$ & No & No \\
\hline Electrochemical $^{30}$ & Trypsin & PBS, artificial serum & $20 \mathrm{fM}$ & $30 \mathrm{~min}$ & Antibody capture & No \\
\hline Fluorescence $^{13}$ & Gastricsin & Pancreatic cyst fluid & $10 \mathrm{nM}$ & $1 \mathrm{hr}$ & No & No \\
\hline Fluorescence $^{79}$ & Prostate Specific Antigen & seminal fluid & $8 \mathrm{nM}$ & $15 \mathrm{~min}$ & No & No \\
\hline Fluorescence $^{62}$ & Proteinase 3 & sputum & $260 \mathrm{pM}$ & $10 \mathrm{~min}$ & Inhibitor capture & Yes \\
\hline Liquid crystal $^{33}$ & Trypsin, Chymotrypsin & PBS & $40 \mathrm{pM}, 4 \mathrm{pM}$ & $3 \mathrm{hr}$ & No & No \\
\hline SERS $^{32}$ & Trypsin & PBS & $1 \mathrm{nM}$ & $5 \mathrm{hr}$ & Gold nanoparticles & No \\
\hline $\begin{array}{l}\text { Magnetic (this } \\
\text { work) }\end{array}$ & Papain & PBS, urine & $4 \mathrm{nM}, 20 \mathrm{nM}$ & $\sim 3.5 \mathrm{~min}$ & No & No \\
\hline
\end{tabular}

Table 1. Comparison between several protease-based assays developed and this work.

potentially quantify various bacterial or fungal proteases present in urine of patients with urinary tract infections by developing a GMR SV sensor assay containing peptide substrates that are specifically cleaved by the microbial proteases. Using a papain concentration of $4 \mathrm{nM}$, we were able to detect protease activity in $3.5 \mathrm{~min}$ and therefore this assay has the potential to rapidly diagnose urinary tract infections, an important parameter for POC device usage. A magnetic detection strategy leverages the inherent negligible background signal in a biofluid which will allow us to achieve comparable sensitivity in serum, sputum, cyst fluid, semen, and wound fluid as urine.

The novel approach reported here has the potential to address several limitations posed by current protease sensors that have been described. Table 1 shows a comparison between this study and other protease detection strategies based on the buffer and/or biofluid tested, sensitivity, assay time, and sample preparation. Other detection methods such as electrochemical and SERS sensors have design requirements for the peptide substrate such that charged amino acids have to be included for optimal detection ${ }^{29,32}$. Peptides used in SPR sensors must have uncharged amino acids to minimize background signal due to their sensitivity to surface charge ${ }^{31}$. Fluorescent and colorimetric protease assays have been developed to detect protease activity in biofluids such as urine ${ }^{57,58}$, cyst fluid $^{13}$, serum ${ }^{59,60}$, semen ${ }^{61}$, and sputum ${ }^{62}$ as well as used to discover protease inhibitors that have subsequently been developed into drugs ${ }^{63-67}$. However, many of these detection modalities require laboratory equipment, and thus are not suitable for POC and POU applications. GMR SV sensor arrays are compatible with complementary metal-oxide-semiconductor (CMOS) technology which can allow them to be inexpensively mass produced ${ }^{42}$ in a disposable format that is amenable to daily use, as well as integration into smartphone-based POC applications ${ }^{68-70}$. The sensor can also be produced in a pre-assembled manner by freeze-drying or lyophilizing the complexes such that it is in a "sample-to-answer" format without the need of additional detection reagents used in traditional ELISA assays. Real-time quantification of protease activity using GMR SV sensor-based detection has significant advantages over current POC protease test kits that detect elevated protease activity in wound fluid ${ }^{71}$ or sputum ${ }^{72}$.

Peptide substrates that are designed to be cleaved by a target protease are often not selective for that enzyme. For example, Miller and colleagues showed that peptide substrates developed for select metalloproteinases, are efficiently cleaved by other proteases ${ }^{73}$. In addition, the peptide cleavage pattern generated by proteases in complex biological samples can be difficult to deconvolute due to overlapping substrate specificity profiles of proteases $^{74}$. Therefore, it is essential to design peptide substrates that are selectively cleaved by the target proteases. Previously, we have identified peptide substrates that are efficiently cleaved by neutrophil elastase in neutrophil protein extracts ${ }^{75}$. Upon immunodepletion of this enzyme from the extract, these peptides were no longer cleaved, thereby validating the selectivity of the substrates for neutrophil elastase. When designing substrates for the GMR SV sensor assay, we will need to take the same approach and identify sequences that are cleaved by the target enzyme with little or no hydrolysis by other proteases in the sample.

The long-term goal of this research is to show that GMR SV sensor-based detection can provide a POC platform to detect various disease related proteases in patient samples. Future peptide sensors will explore the use of alternative peptide substrates that are selectively cleaved by either human or microbial protease targets. In addition, if multiple different peptide substrates provide a more accurate readout for a particular disease, then these substrates can be multiplexed on a single GMR SV chip to potentially quantify activity of multiple proteases simultaneously.

\section{Methods}

Peptide/Linker immobilization on optical plates. Biotin-PEG ${ }_{36}$-Thr-Phe-Ser-Tyr-Nle-Arg-Trp-Pro$\mathrm{PEG}_{12}$-Cys (known as peptide) was synthesized by CPC Scientific and Biotin-PEG-SH (known as linker) was purchased from NANOCS. Peptide $(2 \mu \mathrm{M})$ and linker $(128 \mu \mathrm{M})$ were prepared in $0.1 \mathrm{M} \mathrm{Na}_{2} \mathrm{HPO}_{4}, \mathrm{pH} 7.4,0.15 \mathrm{M}$ $\mathrm{NaCl}, 10 \mathrm{mM}$ EDTA (binding buffer) and added to maleimide activated wells of a 96 -well plate (Pierce catalog $\# 15153$ ) and incubated for $2 \mathrm{~h}$. All wells were then blocked with $57 \mu \mathrm{M}$ Cysteine-HCl (Pierce) in D-PBS for $1 \mathrm{~h}$ followed by $1 \%$ BSA in D-PBS for $1 \mathrm{~h}$. Wells were washed extensively with $0.1 \mathrm{M} \mathrm{Na}_{2} \mathrm{HPO}_{4}, \mathrm{pH} 7.2,0.15 \mathrm{M} \mathrm{NaCl}$, 0.05\% Tween-20 (buffer A) before and after each step. All steps were performed at room temperature.

Blocking with streptavidin on optical plates. After peptide/linker immobilization on maleimide coated wells, background fluorescence in each well was measured in a microplate reader with an excitation of $360 \mathrm{~nm}$ and 
an emission of $460 \mathrm{~nm} .100 \mu \mathrm{L}$ of $5 \mu \mathrm{g} / \mathrm{mL}$ streptavidin, Marina Blue conjugate (ThermoFisher Scientific \#S11221) in D-PBS or $20 \mu \mathrm{g} / \mathrm{mL}$ streptavidin (ThermoFisher Scientific \#21122) in D-PBS was added to wells and incubated for $1 \mathrm{~h}$. Wells were washed extensively with buffer A and then incubated with $100 \mu \mathrm{L}$ of $5 \mu \mathrm{g} / \mathrm{mL}$ streptavidin, Marina Blue conjugate, or $20 \mu \mathrm{g} / \mathrm{mL}$ of streptavidin for $1 \mathrm{~h}$. After washing, $100 \mu \mathrm{L}$ of D-PBS was added to each well and fluorescence was measured. All steps were performed at room temperature. The background fluorescence quantified in each well prior to addition of streptavidin was subtracted from the total fluorescence after addition of streptavidin.

Time-dependent digestion on optical plates. $20 \mathrm{nM}$ of papain in D-PBS, $2 \mathrm{mM}$ DTT was added to each well and the reaction was incubated at room temperature. After defined time intervals of $0,2.5,5,10,15,20$, and 30 mins, the reaction was stopped by addition of E- 64 to a final concentration of $20 \mu \mathrm{M}$. Wells were washed extensively with buffer A, hydrated with $100 \mu \mathrm{L}$ of D-PBS, and fluorescence was measured as outlined above.

Percent reduction calculations for optical assays. To calculate the reduction of fluorescent signal, the background fluorescence $[\mathrm{A}]$ is subtracted from both the pre-assay $[\mathrm{B}]$ and post-assay [C] fluorescence; divided by the difference between $[\mathrm{A}]$ and $[\mathrm{B}]$ and expressed as a percentage.

$$
\% \text { reduction }=\frac{(B-A)-(C-A)}{B-A} \times 100
$$

Conjugation of maleimide activated BSA to peptide and linker. $\quad 20 \mu \mathrm{L}$ of $1.33 \mathrm{mg} / \mathrm{mL}$ solution of BSA-maleimide (Imject Maleimide-Activated BSA, ThermoFisher Scientific \#77115) in water, $56 \mu \mathrm{L}$ of $273 \mu \mathrm{M}$ peptide or $700 \mu \mathrm{M}$ linker, and $24 \mu \mathrm{L}$ of binding buffer were incubated for $24 \mathrm{~h}$ at $4{ }^{\circ} \mathrm{C}$ then dried in a vacuum centrifuge. This complex was then dissolved in $10 \mu \mathrm{L}$ of water.

Chip functionalization. The chip was placed in a UV ozone cleaner for 10 minutes. A custom 3-D printed structure (printed using a Zortrax) with an attached silicone O-ring (High-Temperature High-Purity Silicone O-Rings for Tube Fittings with Straight-Thread Connection, McMaster-Carr \#9396K422) was then screwed onto the chip to allow reagents to be pipetted onto the surface without leakage (Fig. S1a). The chip was washed with $100 \mu \mathrm{L}$ water and $1 \mu \mathrm{L}$ of BSA $(5 \%)$, BSA-peptide $(1.33 \mathrm{mg} / \mathrm{mL}), 1.33 \mathrm{mg} / \mathrm{mL}$ BSA-Linker) was nanospotted onto the corners of the chip sensors with some separation. The chip was then incubated at $4{ }^{\circ} \mathrm{C}$ in a humidity chamber for $24 \mathrm{~h}$, washed with water, and then blocked with $100 \mu \mathrm{L} 5 \%$ BSA for 30 minutes.

Magnetic chip reader. The measurement setup consists of a computer, a power amplifier (PA, Kepco 20-5D), a Helmholtz coil and custom readout electronics ${ }^{76}$. The GMR SV sensor chip, which has 80 individually addressable sensors with a nominal resistance of $1.3 \mathrm{k} \Omega$ and an MR ratio of $10.6 \%$, was placed firmly inside of the Helmholtz coil using a custom designed sensor holder (Fig. S1b). A double modulation readout scheme was used to reject $1 / f$ noise from both the sensors and electronics, and temperature compensation technique was used to reduce temperature drift ${ }^{77}$. The computer digitally adjusted the frequencies and amplitudes of sensor bias voltage and magnetic field through a National Instruments data acquisition card (DAQ, NI PCIe-6351) and a LabVIEW graphical user interface (GUI). Specifically, the PA controlled by the computer provided current into the Helmholtz coil, which offers homogenous magnetic field (23-34 Oe $\mathrm{rms}_{\mathrm{s}}$ based on the sensor MR) for the sensor chip. The readout electronics contain $8 \times$ transimpedance amplifiers to convert the currents to voltages that was quantized by the DAQ. Time-multiplexing was applied to read out the entire $8 \times 10$ sensor array with a 10 second update rate.

Magnetic sensor assays. $\quad 1 \mu \mathrm{L}$ of $1 \mathrm{mg} / \mathrm{mL}$ streptavidin was nanospotted onto sensors containing BSA, BSA-peptide, or BSA-linker and incubated for 30 minutes followed by washing with water and then PBS. The chip was then connected to the electromagnet measurement station (Fig. S1b). Sensors were loaded with $60 \mu \mathrm{L}$ of $50 \mathrm{~nm}$ magnetic nanoparticles coated with streptavidin (Miltenyi Biotec \#130-048-101) for 35 minutes followed by five washes with $100 \mu \mathrm{L}$ buffer A. $100 \mu \mathrm{L}$ of $1 \mathrm{mM}$ biotin (Fisher Scientific, 15486) was added for 10 minutes followed by five washes with $100 \mu \mathrm{L}$ buffer A. $100 \mu \mathrm{L}$ of papain in D-PBS, pH 7.4 or D-PBS, pH 6.0 (adjusted to pH 6.0 with citric acid) was added and incubated for up to 200 minutes. To stop the assay, the sensor was washed five times in buffer A.

Papain activity in urine. Urine from healthy donors was purchased from Innovative Research, Novi, Michigan (IRHUURES50ML). $97 \mu \mathrm{L}$ of urine was incubated with $1 \mu \mathrm{L}$ of human cathepsin B (R\&D Systems, 953-CY-010) and $1 \mu \mathrm{L}$ of DTT for 15 minutes to inactivate the urine cysteine protease inhibitor, cystatin C. The cystatin C-inactivated urine was then added to the sensor well and magnetoresistance was evaluated for $20 \mathrm{~min}$ utes. $1 \mu \mathrm{L}$ of papain was then added to the urine and MR signal was evaluated for 100 minutes. The final concentration of cathepsin B, DTT, and papain in the urine assay was $10 \mu \mathrm{M}, 2 \mathrm{mM}$, and $10 \mathrm{nM}$, respectively.

Magnetic assay time of detection of papain activity calculations. The signal-to-noise ratio (SNR) for each time point was determined by calculating the difference of mean signal (S) for the peptide and linker sensors divided by the noise which is equal to square root of the sum of variances $\left(\sigma^{2}\right)$ of peptide and linker data.

$$
S N R=\frac{S_{\text {peptide }}-S_{\text {linker }}}{\sqrt{\sigma_{\text {peptide }}^{2}+\sigma_{\text {linker }}^{2}}}
$$


Received: 31 January 2020; Accepted: 18 March 2020;

Published online: 14 May 2020

\section{References}

1. Whitcomb, D. C. \& Lowe, M. E. Human pancreatic digestive enzymes. Dig. Dis. Sci. 52, 1-17 (2007).

2. Janis, J. E. \& Attinger, C. E. The basic science of wound healing. Plast. Reconstr. Surg. 117, 12S-34S (2006).

3. Heutinck, K. M., ten Berge, I. J., Hack, C. E., Hamann, J. \& Rowshani, A. T. Serine proteases of the human immune system in health and disease. Mol. Immunol. 47, 1943-1955 (2010).

4. Hochstrasser, M. Ubiquitin, proteasomes, and the regulation of intracellular protein degradation. Curr. Opin. Cell Biol. 7, 215-223 (1995).

5. Duan, G. \& Walther, D. The Roles of Post-translational Modifications in the Context of Protein Interaction Networks. PLoS Comput. Biol. 11 (2015).

6. López-Otín, C. \& Bond, J. S. Proteases: multifunctional enzymes in life and disease. J. Biol. Chem. (2008).

7. Martinelli, P. \& Rugarli, E. I. Emerging roles of mitochondrial proteases in neurodegeneration. Biochim. Biophys. Acta 1797, 1-10 (2010).

8. Trindade, F., Ferreira, R., Amado, F. \& Vitorino, R. Biofluid proteases profiling in diabetes mellitus. Adv. Clin. Chem. 69, 161-207 (2015).

9. Musante, L. et al. Proteases and protease inhibitors of urinary extracellular vesicles in diabetic nephropathy. J. Diabetes Res. 2015, 289734 (2015).

10. Stamey, T. A. et al. Prostate-Specific Antigen as a Serum Marker for Adenocarcinoma of the Prostate. N. Engl. J. Med. 317, 909-916 (1987).

11. Sabbagh, B. et al. Functional protease profiling for laboratory based diagnosis of invasive aspergillosis. Int. J. Oncol. 47, 143-150 (2015).

12. Findeisen, P. \& Neumaier, M. Functional protease profiling for diagnosis of malignant disease. Proteomics Clin. Appl. 6, 60-78 (2012).

13. Ivry, S. L. et al. Global Protease Activity Profiling Provides Differential Diagnosis of Pancreatic Cysts. Clin. Cancer Res. 23, 4865-4874 (2017).

14. MacNee, W. Pathogenesis of Chronic Obstructive Pulmonary Disease. Proc. Am. Thorac. Soc. 2, 258-266 (2005).

15. Barnes, P. J., Shapiro, S. D. \& Pauwels, R. A. Chronic obstructive pulmonary disease: molecular and cellularmechanisms. Eur. Respir. J. 22, 672-688 (2003).

16. Voynow, J. A., Fischer, B. M. \& Zheng, S. Proteases and cystic fibrosis. Int. J. Biochem. Cell Biol. 40, 1238-1245 (2008).

17. Birrer, P. et al. Protease-antiprotease imbalance in the lungs of children with cystic fibrosis. Am. J. Respir. Crit. Care Med. 150, 207-213 (1994)

18. Quinn, R. A. et al. Neutrophilic proteolysis in the cystic fibrosis lung correlates with a pathogenic microbiome. Microbiome 7, 23 (2019).

19. Guo, S. \& DiPietro, L. A. Factors Affecting Wound Healing. J. Dent. Res. 89, 219-229 (2010).

20. McCarty, S. M. \& Percival, S. L. Proteases and Delayed Wound Healing. Adv. Wound Care 2, 438-447 (2013).

21. Warren, A. D., Kwong, G. A., Wood, D. K., Lin, K. Y. \& Bhatia, S. N. Point-of-care diagnostics for noncommunicable diseases using synthetic urinary biomarkers and paper microfluidics. Proc. Natl. Acad. Sci. USA 111, 3671-3676 (2014).

22. Olson, E. S. et al. Activatable cell penetrating peptides linked to nanoparticles as dual probes for in vivo fluorescence and MR imaging of proteases. Proc. Natl. Acad. Sci. USA 107, 4311-4316 (2010).

23. Ntziachristos, V., Tung, C.-H., Bremer, C. \& Weissleder, R. Fluorescence molecular tomography resolves protease activity in vivo. Nat. Med. 8, 757-761 (2002).

24. Chen, C.-H. et al. Enhancing Protease Activity Assay in Droplet-Based Microfluidics Using a Biomolecule Concentrator. J. Am. Chem. Soc. 133, 10368-10371 (2011).

25. Mac, Q. D. et al. Non-invasive early detection of acute transplant rejection via nanosensors of granzyme-B activity. Nat. Biomed. Eng. 3, 281-291 (2019).

26. Ong, I. L. H. \& Yang, K.-L. Recent developments in protease activity assays and sensors. The Analyst 142, 1867-1881 (2017).

27. Parks, T. D., Leuther, K. K., Howard, E. D., Johnston, S. A. \& Dougherty, W. G. Release of proteins and peptides from fusion proteins using a recombinant plant virus proteinase. Anal. Biochem. 216, 413-417 (1994).

28. Callahan, B. P., Stanger, M. J. \& Belfort, M. Protease activation of split green fluorescent protein. Chembiochem Eur. J. Chem. Biol. 11, 2259-2263 (2010).

29. Cao, Y., Yu, J., Bo, B., Shu, Y. \& Li, G. A simple and general approach to assay protease activity with electrochemical technique. Biosens. Bioelectron. 45, 1-5 (2013).

30. Park, S., Kim, G., Seo, J. \& Yang, H. Ultrasensitive Protease Sensors Using Selective Affinity Binding, Selective Proteolytic Reaction, and Proximity-Dependent Electrochemical Reaction. Anal. Chem. 88, 11995-12000 (2016).

31. Chen, H. et al. Potassium ion sensing using a self-assembled calix[4]crown monolayer by surface plasmon resonance. Sens. Actuators B Chem. 133, 577-581 (2008).

32. Yang, L. et al. SERS determination of protease through a particle-on-a-film configuration constructed by electrostatic assembly in an enzymatic hydrolysis reaction. RSC Adv. 6, 90120-90125 (2016).

33. Chen, C.-H. \& Yang, K.-L. Oligopeptide immobilization strategy for improving stability and sensitivity of liquid-crystal protease assays. Sens. Actuators B Chem. 204, 734-740 (2014).

34. McFadyen, I. R., Fullerton, E. E. \& Carey, M. J. State-of-the-Art Magnetic Hard Disk Drives. MRS Bull. 31, 379-383 (2006).

35. Chappert, C., Fert, A. \& Van Dau, F. N. The emergence of spin electronics in data storage. In Nanoscience and Technology 147-157, https://doi.org/10.1142/9789814287005_0015 (Co-Published with Macmillan Publishers Ltd, UK, 2009).

36. Spong, J. K., Speriosu, Fontana, R. E., Dovek, M. M. \& Hylton, T. L. Giant magnetoresistive spin valve bridge sensor. IEEE Trans. Magn. 32, 366-371 (1996).

37. Pelegri, J. et al. A novel spin valve bridge sensor for current sensing. In IMTC 2001. Proceedings of the 18th IEEE Instrumentation and Measurement Technology Conference. Rediscovering Measurement in the Age of Informatics (Cat. No.01CH 37188) vol. $1422-424$ vol.1 (2001).

38. Daughton, J. M. Magnetic tunneling applied to memory (invited). J. Appl. Phys. 81, 3758-3763 (1997).

39. Baselt, D. R. et al. A biosensor based on magnetoresistance technology1This paper was awarded the Biosensors \& Bioelectronics Award for the most original contribution to the Congress.1. Biosens. Bioelectron. 13, 731-739 (1998).

40. Graham, D. L., Ferreira, H. A. \& Freitas, P. P. Magnetoresistive-based biosensors and biochips. Trends Biotechnol. 22, 455-462 (2004).

41. Parkin, S. S. P. Origin of enhanced magnetoresistance of magnetic multilayers: Spin-dependent scattering from magnetic interface states. Phys. Rev. Lett. 71, 1641-1644 (1993).

42. Gani, A. W. et al. An Automated, Quantitative, and Multiplexed Assay Suitable for Point-of-Care Hepatitis B Virus Diagnostics. Sci. Rep. 9 (2019).

43. Aubin, J. E. Autofluorescence of viable cultured mammalian cells. J. Histochem. Cytochem. Off. J. Histochem. Soc. 27, 36-43 (1979). 
44. Giloh, H. \& Sedat, J. W. Fluorescence Microscopy: Reduced Photobleaching of Rhodamine and Fluorescein Protein Conjugates by n-Propyl Gallate. Science 217, 1252-1255 (1982).

45. Lee, J.-R. et al. Multiplex giant magnetoresistive biosensor microarrays identify interferon-associated autoantibodies in systemic lupus erythematosus. Sci. Rep. 6, 27623 (2016).

46. Gaster, R. S. et al. Matrix-insensitive protein assays push the limits of biosensors in medicine. Nat. Med. 15, 1327-1332 (2009).

47. Osterfeld, S. J. et al. Multiplex protein assays based on real-time magnetic nanotag sensing. Proc. Natl. Acad. Sci. 105, 20637-20640 (2008).

48. Costa, A. G., Cusano, N. E., Silva, B. C., Cremers, S. \& Bilezikian, J. P. Cathepsin K: its skeletal actions and role as a therapeutic target in osteoporosis. Nat. Rev. Rheumatol. 7, 447-456 (2011).

49. Choe, Y. et al. Development of $\alpha$-keto-based inhibitors of cruzain, a cysteine protease implicated in Chagas disease. Bioorg. Med. Chem. 13, 2141-2156 (2005).

50. Kim, M. J. et al. Crystal structure of papain-E64-c complex. Binding diversity of E64-c to papain S2 and S3 subsites. Biochem. J. 287, 797-803 (1992)

51. Lapek, J. D. et al. Quantitative Multiplex Substrate Profiling of Peptidases by Mass Spectrometry. Mol. Cell. Proteomics MCP, https:// doi.org/10.1074/mcp.TIR118.001099 (2019).

52. Welch, A. A., Mulligan, A., Bingham, S. A. \& Khaw, K.-T. Urine $\mathrm{pH}$ is an indicator of dietary acid-base load, fruit and vegetables and meat intakes: results from the European Prospective Investigation into Cancer and Nutrition (EPIC)-Norfolk population study. $\mathrm{Br}$. J. Nutr. 99, 1335-1343 (2008).

53. Najeeb, S. et al. Comparison of Urine Dipstick Test with Conventional Urine Culture in Diagnosis of Urinary Tract. Infection. 25, 3 (2015).

54. Taylor, J. M. et al. Aminopeptidase Activities as Prospective Urinary Biomarkers for Bladder Cancer. Proteomics Clin. Appl. 8, 317-326 (2014).

55. Nienhouse, V. et al. Interplay between bladder microbiota and urinary antimicrobial peptides: mechanisms for human urinary tract infection risk and symptom severity. PloS One 9, e114185 (2014).

56. Loomes, L. M., Senior, B. W. \& Kerr, M. A. Proteinases of Proteus spp.: purification, properties, and detection in urine of infected patients. Infect. Immun. 60, 2267-2273 (1992).

57. Poon, C.-Y. et al. FRET-based modified graphene quantum dots for direct trypsin quantification in urine. Anal. Chim. Acta 917, 64-70 (2016).

58. Zhao, L. et al. Fluorescent Strips of Electrospun Fibers for Ratiometric Sensing of Serum Heparin and Urine Trypsin. ACS Appl. Mater. Interfaces 9, 3400-3410 (2017).

59. Streng, A. S. et al. Development of a targeted selected ion monitoring assay for the elucidation of protease induced structural changes in cardiac troponin T. J. Proteomics 136, 123-132 (2016).

60. Lin, T. et al. A sensitive colorimetric assay for cholesterol based on the peroxidase-like activity of MoS2 nanosheets. Microchim. Acta 184, 1233-1237 (2017).

61. Etheridge, T., Straus, J., Ritter, M. A., Jarrard, D. F. \& Huang, W. Semen AMACR protein as a novel method for detecting prostate cancer. Urol. Oncol. Semin. Orig. Investig. 36, 532.e1-532.e7 (2018).

62. Ferguson, T. E. G. et al. P111 Quantification of active proteinase 3 in sputum samples using a novel activity-based immunoassay. Thorax 73, A162-A162 (2018).

63. Matayoshi, E. D., Wang, G. T., Krafft, G. A. \& Erickson, J. Novel fluorogenic substrates for assaying retroviral proteases by resonance energy transfer. Science 247, 954-958 (1990).

64. Kisselev, A. F. \& Goldberg, A. L. Monitoring Activity and Inhibition of 26S Proteasomes with Fluorogenic Peptide Substrates. In Methods in Enzymology vol. 398 364-378 (Academic Press, 2005).

65. Moss, M. L. \& Rasmussen, F. H. Fluorescent substrates for the proteinases ADAM17, ADAM10, ADAM8, and ADAM12 useful for high-throughput inhibitor screening. Anal. Biochem. 366, 144-148 (2007).

66. Lai, K. S., Ho, N.-H., Cheng, J. D. \& Tung, C.-H. Selective Fluorescence Probes for Dipeptidyl Peptidase Activity - Fibroblast Activation Protein and Dipeptidyl Peptidase IV. Bioconjug. Chem. 18, 1246-1250 (2007).

67. Watzke, A. et al. Selective activity-based probes for cysteine cathepsins. Angew. Chem. Int. Ed Engl. 47, 406-409 (2008).

68. Choi, J. et al. Portable, one-step, and rapid GMR biosensor platform with smartphone interface. Biosens. Bioelectron. 85, 1-7 (2016).

69. Ng, E., Yao, C., Shultz, T. O., Ross-Howe, S. \& Wang, S. X. Magneto-nanosensor smartphone platform for the detection of HIV and leukocytosis at point-of-care. Nanomedicine Nanotechnol. Biol. Med. 16, 10-19 (2019).

70. Gaster, R. S., Hall, D. A. \& Wang, S. X. nanoLAB: an ultraportable, handheld diagnostic laboratory for global health. Lab. Chip 11, 950-956 (2011)

71. Serena, D. T. et al. Preliminary results: Testing for elevated protease activity in clinical practice. Wound Repair Regen. 20 , A112.

72. McCafferty, D. F. et al. P116 Neatstik - a novel point of care test for the measurement of active neutrophil elastase in patients with respiratory disease. Thorax 72, A146-A146 (2017).

73. Miller, M. A. et al. Proteolytic Activity Matrix Analysis (PrAMA) for Simultaneous Determination of Multiple Protease Activities. Integr. Biol. Quant. Biosci. Nano Macro 3, 422-438 (2011)

74. Villanueva, J. et al. Differential exoprotease activities confer tumor-specific serum peptidome patterns. J. Clin. Invest. 116, 271-284 (2006).

75. O’Donoghue, A. J. et al. Global substrate profiling of proteases in human neutrophil extracellular traps reveals consensus motif predominantly contributed by elastase. PloS One 8, e75141 (2013).

76. Hall, D. A. et al. GMR biosensor arrays: a system perspective. Biosens. Bioelectron. 25, 2051-2057 (2010).

77. Hall, D. A., Gaster, R. S., Osterfeld, S. J., Murmann, B. \& Wang, S. X. GMR Biosensor Arrays: Correction Techniques for Reproducibility and Enhanced Sensitivity. Biosens. Bioelectron. 25, 2177-2181 (2010).

78. Ding, X. \& Yang, K.-L. Enzymatic Deposition of Silver Particles for Detecting Protease Activity. Part. Part. Syst. Charact. 31, 1300-1306 (2014).

79. Denmeade, S. R. et al. Specific and efficient peptide substrates for assaying the proteolytic activity of prostate-specific antigen. Cancer Res. 57, 4924-4930 (1997).

\section{Acknowledgements}

X.Z. and D.A.H. acknowledge funding from the National Science Foundation (Grant ECCS-1454608). The research was supported in part by the National Institutes of Health, Grant UL1TR001442 and EB028485 awarded to A.J.O. and D.A.H.

\section{Author contributions}

S.A., S.J., A.J.O. and D.A.H. conceived the research. S.A., S.J. and M.S. designed and conducted the optical and magnetic assay experiments. X.Z. designed the electronic system and signal processing. S.A., S.J., M.S., X.Z., A.J.O. and D.A.H. wrote the paper. All authors discussed the results and reviewed the manuscript. 


\section{Competing interests}

D.A.H. has related patents or patent applications assigned to Stanford University and out-licensed for potential commercialization. D.A.H. has stock in MagArray, Inc. and Flux Biosciences, which have licensed relevant patents from Stanford University for commercialization of GMR biosensor chips. All other authors have no competing interests to declare.

\section{Additional information}

Supplementary information is available for this paper at https://doi.org/10.1038/s41598-020-62910-2.

Correspondence and requests for materials should be addressed to A.J.O. or D.A.H.

Reprints and permissions information is available at www.nature.com/reprints.

Publisher's note Springer Nature remains neutral with regard to jurisdictional claims in published maps and institutional affiliations.

(c) (i) Open Access This article is licensed under a Creative Commons Attribution 4.0 International License, which permits use, sharing, adaptation, distribution and reproduction in any medium or format, as long as you give appropriate credit to the original author(s) and the source, provide a link to the Creative Commons license, and indicate if changes were made. The images or other third party material in this article are included in the article's Creative Commons license, unless indicated otherwise in a credit line to the material. If material is not included in the article's Creative Commons license and your intended use is not permitted by statutory regulation or exceeds the permitted use, you will need to obtain permission directly from the copyright holder. To view a copy of this license, visit http://creativecommons.org/licenses/by/4.0/.

(c) The Author(s) 2020 